\title{
The Role of MRI in Diagnosing Neurovascular Compression of the Cochlear Nerve Resulting in Typewriter Tinnitus
}

\author{
(D) Y.J. Bae, (D) Y.J. Jeon, (D) B.S. Choi, (D).-W. Koo, and (D).-J. Song
}

\begin{abstract}
BACKGROUND AND PURPOSE: Typewriter tinnitus, a symptom characterized by paroxysmal attacks of staccato sounds, has been thought to be caused by neurovascular compression of the cochlear nerve, but the correlation between radiologic evidence of neurovascular compression of the cochlear nerve and symptom presentation has not been thoroughly investigated. The purpose of this study was to examine whether radiologic evidence of neurovascular compression of the cochlear nerve is pathognomonic in typewriter tinnitus.
\end{abstract}

MATERIALS AND METHODS: Fifteen carbamazepine-responding patients with typewriter tinnitus and 8 control subjects were evaluated with a 3D T2-weighted volume isotropic turbo spin-echo acquisition sequence. Groups 1 (16 symptomatic sides), 2 (14 asymptomatic sides), and 3 (16 control sides) were compared with regard to the anatomic relation between the vascular loop and the internal auditory canal and the presence of neurovascular compression of the cochlear nerve with/without angulation/indentation.

RESULTS: The anatomic location of the vascular loop was not significantly different among the 3 groups (all, $P>.05$ ). Meanwhile, neurovascular compression of the cochlear nerve on MR imaging was significantly higher in group 1 than in group $3(P=.032)$. However, considerable false-positive (no symptoms with neurovascular compression of the cochlear nerve on MR imaging) and false-negative (typewriter tinnitus without demonstrable neurovascular compression of the cochlear nerve) findings were also observed.

CONCLUSIONS: Neurovascular compression of the cochlear nerve was more frequently detected on the symptomatic side of patients with typewriter tinnitus compared with the asymptomatic side of these patients or on both sides of control subjects on MR imaging. However, considering false-positive and false-negative findings, meticulous history-taking and the response to the initial carbamazepine trial should be regarded as more reliable diagnostic clues than radiologic evidence of neurovascular compression of the cochlear nerve.

ABBREVIATIONS: $\mathrm{ABR}=$ abnormal auditory brain stem response; $\mathrm{CPA}=$ cerebellopontine angle; $\mathrm{IAC}=$ internal acoustic canal; $\mathrm{NVC}-\mathrm{C}=$ neurovascular compression of the cochlear nerve; T2-VISTA = T2-weighted volume isotropic turbo spin-echo acquisition

A rterial compression of the cochleovestibular nerve complex has been suggested as a potential cause of hearing deficit, typewriter tinnitus, and equilibrium disturbance or vertigo. ${ }^{1-4}$ Among these clinical symptoms, typewriter tinnitus, which was

Received August 31, 2016; accepted after revision January 25, 2017.

From the Departments of Radiology (Y.J.B., B.S.C.) and Otorhinolaryngology-Head and Neck Surgery (Y.J.., J.-W.K., J.-J.S.), Seoul National University Bundang Hospital, Seongnam, Korea.

This work was supported by a grant from the Korea Health Technology R\&D Project through the Korea Health Industry Development Institute, funded by the Ministry of Health and Welfare, Republic of Korea (grant No. HI14C2264).

The authors declare no conflict of interest related to funding sources or financial holdings that might raise questions about possible sources of bias.

Please address correspondence to Jae-Jin Song, MD, PhD, Department of Otorhinolaryngology-Head \& Neck Surgery, Seoul National University Bundang Hospital, 300 Gumi-dong, Bundang-gu, Seongnam, 13620, Republic of Korea; e-mail: jjsong96@snubh.org or jjsong96@gmail.com

- Indicates open access to non-subscribers at www.ajnr.org

Indicates article with supplemental on-line photo.

http://dx.doi.org/10.3174/ajnr.A5156 first described by a pediatric cardiologist as "ear-clicking tinnitus responding to carbamazepine," ${ }^{5}$ is characterized by paroxysmal attacks. It is either spontaneous or precipitated by positioning or sounds and occurs with staccato sounds described as "Morse code," "machine gun," "coins in a can," "crackling," or "typewriter" sounds. ${ }^{6-8}$ Typewriter tinnitus is considered the result of dysmyelination and demyelination of the contact point between the arterial loop and the cochlear nerve that transmits an abnormal signal to the auditory cortex. ${ }^{9}$ As in other vascular compression syndromes such as trigeminal neuralgia, typewriter tinnitus is highly responsive to carbamazepine. ${ }^{6-8,10,11}$ Complete suppression of tinnitus with carbamazepine treatment, in addition to its paroxysmal character, has led to the hypothesis that typewriter tinnitus results from neurovascular compression of the cochlear nerve (NVC-C), for which microvascular compression would be an effective treatment. ${ }^{6,7}$

However, because typewriter tinnitus is a relatively rare condition and was only recently described, few studies have been performed investigating the relationship between radiologic evi- 

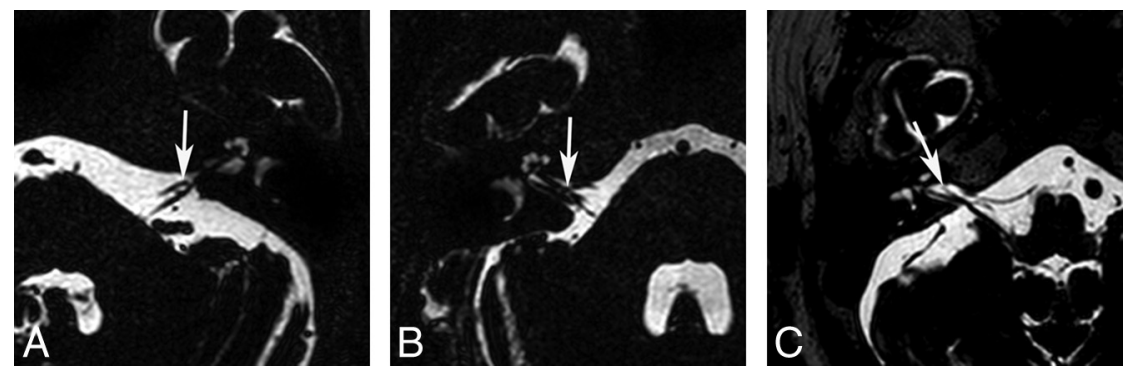

presented with unilateral tinnitus except for 1 patient who had bilateral tinnitus, 2 groups were included in the analysis as follows: group 1 , symptomatic sides $(n=16)$, and group 2, asymptomatic sides $(n=14)$. During the same period, 8 control subjects (4 men and 4 women; age range, 47-74 years; mean age, 60.5 years) who did not have tinnitus but underwent MR imaging for dizziness were also included in our

FIG 1. Types of AICA loops. A, Type I AICA loops lie within the CPA (arrow) but do not enter the IAC. B, Type II AICA loops enter the IAC (arrow) but do not extend into $>50 \%$ of the length of the IAC. C, Type III AICA loops extend into $>50 \%$ of the IAC (arrow).

study (16 sides, group 3). No patient had a history of neurologic disease, tumors in the IAC or CPA, or temporal bone trauma.

dence of cochlear nerve compression on MR imaging and the presence of typewriter tinnitus, to our knowledge. A few previous studies investigating subjects with typewriter tinnitus with carbamazepine responsiveness showed evidence of NVC-C on T2weighted CISS images; however, the sample sizes were relatively small (4 and 5 subjects, respectively), and no control subjects without tinnitus were included. ${ }^{6,10}$ Moreover, signs of neurovascular compression have frequently been detected on MR imaging in asymptomatic patients, which raises questions about the role of MR imaging in the diagnosis of typewriter tinnitus. ${ }^{7,12}$

Thus, in this study, we aimed to evaluate MR imaging findings of subjects with typewriter tinnitus with regard to the presence of radiologic evidence of cochlear nerve compression by performing a 3D T2-weighted volume isotropic turbo spin-echo acquisition (T2-VISTA; Phillips Healthcare, Best, the Netherlands) sequence on 3T MR imaging to effectively visualize neurovascular compression. In other words, the purpose of the current study was to examine whether radiologic evidence of cochlear nerve compression is pathognomonic in subjects with typewriter tinnitus.

\section{MATERIALS AND METHODS Subjects}

This retrospective study was approved by the institutional review board of the Clinical Research Institute of Seoul National Bundang Hospital (B-1608-360-101). From January 2014 to April 2016, 27 patients were initially diagnosed with typewriter tinnitus at our institution. The subjects visited the outpatient clinic of the otorhinolaryngology department with typical symptoms of typewriter tinnitus, which they described as "typewriter," "machine gun," or "crackling" sounds. After we excluded other possible pathologic causes of tinnitus by taking a thorough history and performing neuro-otologic examinations, the patients were prescribed carbamazepine as an initial empiric treatment. Of the 27 subjects, 2 were lost to follow-up and were excluded from further analysis. After a 3-week trial, all 25 patients reported complete resolution or marked improvement of tinnitus.

At the initial visit, MR imaging of the internal acoustic canal (IAC) and the cerebellopontine angle (CPA) was recommended for all patients with typewriter tinnitus. Of the 25 subjects, 15 ( 5 men and 10 women; age range, 27-84 years; mean age, 54.1 years) underwent MR imaging of the IAC and CPA; thus, these 15 subjects were finally enrolled in the current study. Two neuroradiologists (Y.J.B. and B.S.C, with 7 and 17 years of experience, respectively) evaluated both the IACs and CPAs of all patients separately. Because all patients

\section{MR Imaging Protocol}

MR imaging was performed by using a $3 \mathrm{~T}$ instrument (Achieva and Ingenia; Philips Healthcare) with a 32-channel SENSE Head Coil (Philips Healthcare). T2-VISTA imaging of the IAC and CPA was performed with the following parameters: FOV, $160 \times 160$ $\mathrm{mm}^{2}$; acquisition matrix size, $228 \times 228$; section thickness, 0.7 mm; overlapping, 0.35 mm; NEX, 1; TR, 2000 ms; TE, 250 ms; flip angle, $90^{\circ}$. In addition, the following sequences were obtained according to clinical need: axial T2WI of the whole brain (FOV, $185 \times 230 \mathrm{~mm}^{2}$; acquisition matrix size, $420 \times 375$; section thickness, $5 \mathrm{~mm}$; section gap, $1 \mathrm{~mm}$; NEX, 1; TR, $3000 \mathrm{~ms}$; TE, $80 \mathrm{~ms}$; flip angle, $90^{\circ}$ ), axial T1WI of the IAC and CPA (FOV, $180 \times 180$ $\mathrm{mm}^{2}$; acquisition matrix size, $272 \times 217$; section thickness, $3 \mathrm{~mm}$; section gap, $0 \mathrm{~mm}$; NEX, 1; TR, $500 \mathrm{~ms}$; TE, $10 \mathrm{~ms}$; flip angle, 50), and $3 \mathrm{D}$ gadolinium-enhancing T1WI of the IAC and CPA (FOV, $200 \times 200 \mathrm{~mm}^{2}$; acquisition matrix size, $256 \times 256$; section thickness, $1 \mathrm{~mm}$; section gap, $1 \mathrm{~mm}$; NEX, 1; TR, $9.5 \mathrm{~ms}$; TE, $3.3 \mathrm{~ms}$; flip angle, $8^{\circ}$ ).

\section{Imaging Analysis}

Two neuroradiologists blinded to the clinical findings of the subjects evaluated the neurovascular structures of the IAC and CPA on axial 3D T2-VISTA images and sagittal and/or coronal reconstructed images and made final decisions in consensus. First, the type of AICA loop of all study subjects was determined by using the Chavda classification ${ }^{13}$ as follows: type I, the AICA loop lying within the CPA but not entering the IAC (Fig $1 A$ and On-line Fig $1 A$ ); type II, the AICA loop entering the IAC but not extending $>50 \%$ of the length of the IAC (Fig $1 B$ and On-line Fig $1 B$ ); and type III, the AICA loop extending into $>50 \%$ of the IAC (Fig $1 C$ and On-line Fig 1C). Second, the type of neurovascular contact was classified into the following 3 categories: type I, no neurovascular contact (Fig $2 A$ and On-line Fig 2A); type II, neurovascular contact present at the cochleovestibular nerve complex but without angulation/indentation of the nerve (Fig $2 B$ and On-line Fig $2 B$ ); and type III, neurovascular compression causing cochleovestibular nerve angulation/indentation (Fig $2 \mathrm{C}$ and On-line Fig $2 C) .{ }^{12}$ In addition, the presence of neurovascular contact with arterial structures other than the AICA was assessed.

\section{Statistical Analysis}

The type of AICA loop and the type of neurovascular contact on MR imaging in groups 1 and 2 were compared by using the Wilcoxon signed rank test for paired, nonparametric, ordinal data. 

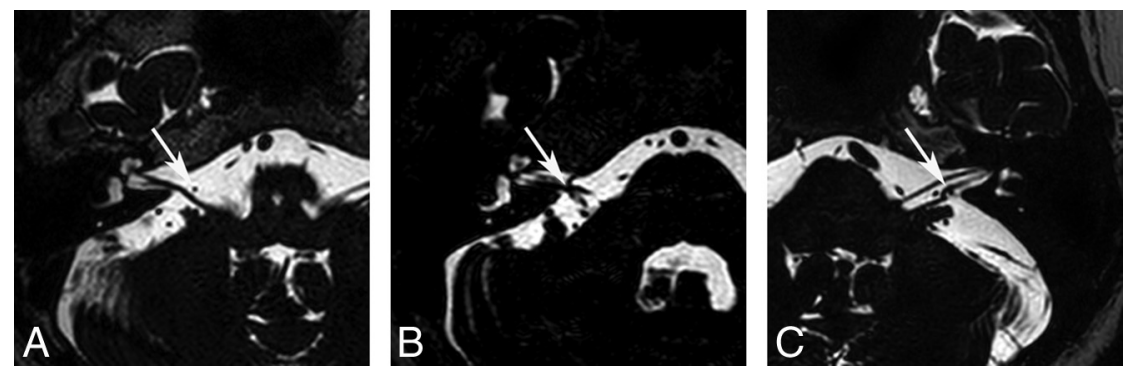

FIG 2. Neurovascular contact types between the AICA and the cochleovestibular nerve. A, A type I neurovascular contact shows no neurovascular contact (arrow). B, A type II neurovascular contact shows contact (arrow) between the AICA and the cochleovestibular nerve without angulation/indentation of the nerve. C, A type III neurovascular contact shows angulation/ indentation (arrow) of the cochleovestibular nerve by the AICA loop.

MRI findings according to patient groups

\begin{tabular}{ccccc}
\hline Type & $\begin{array}{c}\text { Group 1 } \\
(\boldsymbol{n}=16)\end{array}$ & $\begin{array}{c}\text { Group 2 } \\
(\boldsymbol{n}=14)\end{array}$ & $\begin{array}{c}\text { Group 3 } \\
(\boldsymbol{n}=16)\end{array}$ & P Values \\
\hline AICA & & & & \\
I & $7(43.8 \%)$ & $7(50.0 \%)$ & $13(81.3 \%)$ & \\
II & $7(43.8 \%)$ & $6(42.9 \%)$ & $2(12.5 \%)$ & \\
III & $2(12.5 \%)$ & $1(7.1 \%)$ & $1(6.3 \%)$ &
\end{tabular}

Neurovascular $.803^{\mathrm{a}} / .158^{\mathrm{b}} / .065^{\mathrm{c}}$

Neurovascular
contact

$\begin{array}{llll}\text { I } & 5(31.3 \%) & 8(57.1 \%) & 10(62.5 \%) \\ \text { II } & 8(50.0 \%) & 6(42.9 \%) & 6(37.5 \%) \\ \text { III } & 3(18.8 \%) & 0(0 \%) & 0(0 \%)\end{array}$

Wilcoxon signed rank test.

${ }^{\mathrm{b}} P$ values indicate the results of statistical comparisons between groups 2 and 3 by the linear association test.

c $P$ values indicate the results of statistical comparisons between groups 1 and 3 by the linear association test.

${ }^{\mathrm{d}} P$ values $<.05$

The comparisons between groups 2 and 3 and between groups 1 and 3 were performed by the linear association test for independent, nonparametric, ordinal data. A $P$ value $<.05$ indicated statistical significance. All statistical analyses were performed by using SPSS software (Version 18.0; IBM, Armonk, New York).

\section{RESULTS}

The Table summarizes the results of the MR imaging findings of the 3 subject groups.

\section{AICA Loop Type}

Group 1 (16 symptomatic sides of subjects with typewriter tinnitus) showed 7 type I (43.8\%), 7 type II (43.8\%), and 2 type III (12.5\%) AICA loops. Group 2 (14 asymptomatic sides of subjects with typewriter tinnitus) showed 7 type I (50.0\%), 6 type II (42.9\%), and 1 type III (7.1\%) AICA loops. Meanwhile, of the 16 AICA loops of group 3, 13 were type I (81.3\%), 2 were type II $(12.5 \%)$, and 1 was type III $(6.3 \%)$. The comparison of the anatomic location of AICA loops in the IAC and CPA among the 3 groups did not yield statistically significant differences (Table) but showed a trend toward the incidence of a type III AICA loop being higher in group 1 and of a type I AICA loop being higher in group 3.
Types of Neurovascular Contact

Five sides $(31.3 \%)$ in group 1,8 sides $(57.1 \%)$ in group 2 , and 10 sides $(62.5 \%)$ in group 3 showed type I neurovascular contact. Type II neurovascular contact (without nerve angulation/indentation) was shown in 8 sides $(50.0 \%)$ in group 1 , 6 sides $(42.9 \%)$ in group 2, and 6 sides $(37.5 \%)$ in group 3 . Type III neurovascular contact (compression with nerve angulation/indentation) was demonstrated in 3 of 16 sides (18.8\%) in group 1, while no subjects in group 2 or 3 were classified as having type III neurovascular contact. The type of neurovascular contact was significantly different between groups 1 and 3 (Table, $P=.032$ ). Meanwhile, no vascular structure other than the AICA loop was found to contribute to neurovascular contact of the cochleovestibular nerve.

\section{DISCUSSION}

In the current study, we evaluated MR imaging findings of subjects with typewriter tinnitus with regard to the presence of neurovascular cross-compression of the cochlear nerve to investigate whether radiologic evidence of cochlear nerve compression is pathognomonic in typewriter tinnitus. Neurovascular structures are known to be well-visualized on heavily T2-weighted images such as T2-VISTA, FIESTA, or CISS. With T2-VISTA imaging, our results showed that the anatomic location of the AICA loop in the IAC and CPA among the symptomatic and asymptomatic sides of subjects with typewriter tinnitus and controls without tinnitus did not yield statistically significant differences. However, the incidence of neurovascular compression causing angulation/indentation of the cochlear nerve was significantly higher on the symptomatic sides of patients with typewriter tinnitus than in the other groups.

\section{Previous Literature on the Radiologic Findings of Typewriter Tinnitus and the Novelty of the Current Study}

The concept of neurovascular compression syndrome of the cochleovestibular nerve has been continuously proposed since Jannetta et $\mathrm{al}^{14,15}$ first suggested that arterial compression of the cochleovestibular nerve might cause hearing loss, vertigo, and tinnitus. ${ }^{2,12,16-22}$ Many studies have advocated this concept by performing microvascular decompression in patients suspected of having cochleovestibular neurovascular compression syndrome and showing favorable clinical outcomes after the operation..$^{2-4,14,15,17,19,23}$

However, there has also been much controversy regarding the relationship between radiologic evidence of neurovascular compression and audiovestibular symptoms. A major concern is that AICA loops are frequently found in the IAC even in asymptomatic healthy subjects. ${ }^{13,24}$ Additionally, because heavily T2-weighted high-resolution images such as T2-VISTA, FIESTA, and CISS have been used to provide excellent delineation of the neurovascular structures in the IAC and CPA, many radiologic studies have reported a comparable incidence of neurovascular contact be- 
tween the AICA and the cochleovestibular nerve in symptomatic and asymptomatic subjects. ${ }^{20,25,26}$ Indeed, several MR imaging studies have claimed that there was no significant association between the presence of tinnitus and AICA configuration or neurovascular compression. ${ }^{12,21}$ Gultekin et al $^{12}$ compared 58 patients with unexplained tinnitus and 44 controls with regard to the presence of neurovascular contact and the presence of nerve angulation by the vessel with a 3D-FIESTA sequence but concluded that vascular compression of the cochleovestibular nerve could not be a cause of tinnitus.

However, no previous studies have investigated the relationship between MR imaging findings of the AICA loop in the IAC and CPA and the presence of typewriter tinnitus symptoms, to our knowledge. A few studies have enrolled patients with unexplained tinnitus regardless of their symptomatology, but these studies did not perform subgrouping of the subjects with regard to the psychoacoustic characteristics of tinnitus. ${ }^{12,21}$ While nonpulsatile subjective tinnitus is thought to be a result of functional changes in auditory or nonauditory brain areas ${ }^{27-32}$ and responds very poorly to almost all kinds of medical treatment, ${ }^{33}$ typewriter tinnitus is known to have characteristic paroxysmal staccato sounds and is regarded as the result of NVC-C by the AICA loop based on its excellent response to carbamazepine. ${ }^{6-8,10,11}$ Two studies have investigated the MR imaging findings of 4 and 5 subjects with typewriter tinnitus, respectively, with regard to vascular compression of the auditory nerve, ${ }^{6,10}$ but these studies lacked controls. Therefore, MR imaging findings should be assessed only in patients with typewriter tinnitus who showed a quick response to carbamazepine and should be compared with patients with asymptomatic sides or with healthy controls, as in the current study, to properly evaluate the relationship between neurovascular compression and typewriter tinnitus.

\section{MR Imaging May Help in the Diagnosis of NVC-C Resulting in Typewriter Tinnitus}

During 2 years, we enrolled 25 patients diagnosed with typewriter tinnitus and evaluated the types of AICA loops in the IAC and CPA and the types of neurovascular contact by using 3T 3D T2VISTA imaging in 15 patients. When we compared the symptomatic sides of patients with typewriter tinnitus (group 1) with the asymptomatic sides of those with typewriter tinnitus (group 2) and healthy controls (group 3), the difference was not statistically significant (Table). However, considering that in a previous study, type II and type III AICA loops were observed in $38.0 \%$ of 332 patients with ipsilateral auditory symptoms, ${ }^{13}$ our results, which demonstrated that $56.3 \%$ of group 1 and $18.8 \%$ of group 3 had type II and III AICA loops, showed a definite tendency toward type II and III AICA loops on the symptomatic sides of subjects with typewriter tinnitus. Moreover, the 3 groups in this study had significantly different (Table) neurovascular contact types. In group $1,68.8 \%$ of the subjects showed type II or III neurovascular contact, which is comparable with findings in a previous study showing neurovascular contact in 4 of 5 (80\%) patients with typewriter tinnitus. ${ }^{6}$ In this regard, radiologic evidence of the AICA loop entering the IAC and contact between the AICA loop and the vestibulocochlear nerve may be of additive value in diagnosing NVC-C presenting with typewriter tinnitus.
However, there were considerable false-positive and false-negative radiologic findings in our subjects. Indeed, $43.8 \%$ of group 1 showed AICA loops lying within the CPA but not entering the IAC, and $31.3 \%$ did not show neurovascular contact with or without nerve angulation/indentation on T2-VISTA (false-negative results). In contrast, of the 30 sides included in groups 2 and 3, 10 subjects (33.3\%) had AICA loops entering the IAC and 12 subjects $(40.0 \%)$ showed neurovascular contact with or without nerve angulation/indentation (false-positive results). Considering that group 1 comprised subjects with characteristic symptoms and excellent responses to carbamazepine and that groups 2 and 3 were nonsymptomatic subjects and healthy controls, respectively, false-positive and false-negative results in up to $40 \%$ of the study population may reveal the incomplete role of IAC MR imaging for the diagnosis of typewriter tinnitus. In other words, although previous literature on medical and surgical treatment outcomes has proved that typewriter tinnitus originates from neurovascular contact, the current radiologic imaging modalities cannot completely confirm or rule out the presence of typewriter tinnitus. This situation may be attributed to the following assumptions: First, some neurovascular contact may accompany anatomic proximity, but not direct contact, between the AICA loop and the cochlear nerve. In 2 previous studies investigating subjects with typewriter tinnitus, symptoms were triggered by head position changes or loud sounds. Thus, neurovascular contact between the AICA loop and cochlear nerve is "dynamic" in some patients, meaning that the contact is on and off and that MR imaging may have only captured the "contact-off" status. Second, because false-positive results (ie, evidence of neurovascular contact without any symptoms) existed in a considerable percentage of our subjects, symptoms may be elicited not by direct anatomic contact but by demyelination of the cochlear nerve due to the proximity of the AICA loop, regardless of radiologically demonstrable contact between the 2 .

Therefore, we suggest that meticulous history-taking regarding subjectively perceived tinnitus characteristics and their aggravating factors as well as the response to an initial carbamazepine trial should be regarded as more reliable diagnostic clues than radiologic evidence of neurovascular contact in subjects with typewriter tinnitus. However, considering that the symptoms can be cured by microvascular decompression of the cochlear nerve, it may be of value to evaluate all symptomatic patients with typewriter tinnitus with IAC MR imaging, including a heavily T2weighted sequence, to see whether the anatomic change is severe enough to consider surgical treatment.

\section{Limitations of the Current Study and Proposed Future Studies}

To the best of our knowledge, this is the first case-control report to compare the radiologic characteristics of subjects with typewriter tinnitus. However, our case series is limited in several aspects. First, abnormal auditory brain stem response (ABR) findings have been suggested to be diagnostic of NVC-C, ${ }^{19,34}$ but ABR was not tested in our series because most of our patients had a relatively short history and previous literature revealed that patients with a short history of NVC-C usually showed intact ABR results. ${ }^{19,34}$ However, future studies including ABR for the initial 
evaluation of patients should be performed to further evaluate the value of ABR as a diagnostic tool. Second, because we strictly enrolled patients diagnosed with typewriter tinnitus on the basis of their immediate response to a carbamazepine trial, our sample size was relatively small. Therefore, future studies with a larger number of subjects are warranted to further verify our current results. Next, none of the subjects enrolled in the current study underwent microvascular decompression surgery because all subjects reported abated symptoms after carbamazepine treatment. Because decompressing the cochlear nerve and determining symptom relief would be the direct way to confirm the causal relationship between neurovascular contact and typewriter tinnitus, future radiologic studies on subjects treated with microvascular decompression may further reveal the causative relationship, along with evidence from MR imaging findings and the presence of typewriter tinnitus. One subject had bilateral typewriter tinnitus, and some subjects had false-positive findings (ie, radiologic evidence of $\mathrm{NVC}-\mathrm{C}$ ); thus, subjects with unilateral typewriter tinnitus may develop bilateral symptoms in the future. If there are such cases, predicting future symptom development based on radiologic findings will be of further value. Future longitudinal follow-up of the current subjects should be performed to investigate such a possibility.

\section{CONCLUSIONS}

Taken together, our results indicate that NVC-C by the AICA loop was more frequently detected on the symptomatic side of subjects with typewriter tinnitus than on the asymptomatic side of these subjects or on both sides of control subjects on MR imaging. However, because all subjects with typewriter tinnitus were strictly enrolled on the basis of their carbamazepine response, some subjects had typewriter tinnitus without NVC-C, and some controls without tinnitus had radiologic evidence of NVC-C, current MR imaging modalities may only be of additive value for the diagnosis of NVC-C resulting in typewriter tinnitus. In other words, meticulous history-taking and the response to the initial carbamazepine trial should be regarded as more reliable diagnostic clues than radiologic evidence of NVC-C.

\section{ACKNOWLEDGMENTS}

The English in this document has been checked by at least 2 professional editors, both native speakers of English. Please see http:// www.textcheck.com/certificate/eqogwz for a certificate. The authors thank the Medical Research Collaborating Center at Seoul National University Bundang Hospital for consultation on the statistical analyses.

\section{REFERENCES}

1. Jannetta PJ. Cranial nerve vascular compression syndromes (other than tic douloureux and hemifacial spasm). Clin Neurosurg 1981;28: 445-56 Medline

2. Yap L, Pothula VB, Lesser T. Microvascular decompression of cochleovestibular nerve. Eur Arch Otorhinolaryngol 2008;265:861-69 CrossRef Medline

3. Guevara N, Deveze A, Buza V, et al. Microvascular decompression of cochlear nerve for tinnitus incapacity: pre-surgical data, surgical analyses and long-term follow-up of $\mathbf{1 5}$ patients. Eur Arch Otorhinolaryngol 2008;265:397-401 CrossRef Medline

4. Moller MB, Moller AR, Jannetta PJ, et al. Microvascular decompres- sion of the eighth nerve in patients with disabling positional vertigo: selection criteria and operative results in 207 patients. Acta Neurochir (Wien) 1993;125:75-82 CrossRef Medline

5. Mardini MK. Ear-clicking "tinnitus" responding to carbamazepine. N Engl J Med 1987;317:1542 Medline

6. Levine RA. Typewriter tinnitus: a carbamazepine-responsive syndrome related to auditory nerve vascular compression. ORL J Otorhinolaryngol Relat Spec 2006;68:43-46; discussion 46-47 CrossRef Medline

7. Mathiesen T, Brantberg K. Microvascular decompression for typewriter tinnitus-case report. Acta Neurochir (Wien) 2015;157:333-36 CrossRef Medline

8. Nam EC, Handzel O, Levine RA. Carbamazepine responsive typewriter tinnitus from basilar invagination. J Neurol Neurosurg Psychiatry 2010;81:456-58 CrossRef Medline

9. De Ridder D, Ryu H, Moller AR, et al. Functional anatomy of the human cochlear nerve and its role in microvascular decompressions for tinnitus. Neurosurgery 2004;54:381-88; discussion 388-90 CrossRef Medline

10. Brantberg K. Paroxysmal staccato tinnitus: a carbamazepine responsive hyperactivity dysfunction symptom of the eighth cranial nerve. J Neurol Neurosurg Psychiatry 2010;81:451-55 CrossRef Medline

11. Salvi R, Lobarinas E, Sun W. Pharmacological treatments for tinnitus: new and old. Drugs Future 2009;34:381-400 CrossRef Medline

12. Gultekin S, Celik H, Akpek S, et al. Vascular loops at the cerebellopontine angle: is there a correlation with tinnitus? AJNR Am J Neuroradiol 2008;29:1746-49 CrossRef Medline

13. McDermott AL, Dutt SN, Irving RM, et al. Anterior inferior cerebellar artery syndrome: fact or fiction. Clin Otolaryngol Allied Sci 2003; 28:75-80 CrossRef Medline

14. Jannetta PJ. Neurovascular cross-compression in patients with hyperactive dysfunction symptoms of the eighth cranial nerve. Surg Forum 1975;26:467-69 Medline

15. Jannetta PJ. Outcome after microvascular decompression for typical trigeminal neuralgia, hemifacial spasm, tinnitus, disabling positional vertigo, and glossopharyngeal neuralgia (honored guest lecture). Clin Neurosurg 1997;44:331-83 Medline

16. Gierek T, Markowski J, Majzel K, et al. Disabling positional vertigo (DPV): syndrome of vestibulo-cochlear organ impairment during vascular compression of the vestibulo-cochlear nerve (VCS) [in Polish]. Otolaryngol Pol 2005;59:403-07 Medline

17. Brackmann DE, Kesser BW, Day JD. Microvascular decompression of the vestibulocochlear nerve for disabling positional vertigo: the House Ear Clinic experience. Otol Neurotol 2001;22:882-87 CrossRef Medline

18. Markowski J, Gierek T, Kluczewska E, et al. Assessment of vestibulocochlear organ function in patients meeting radiologic criteria of vascular compression syndrome of vestibulocochlear nervediagnosis of disabling positional vertigo. Med Sci Monit 2011;17: CR169-73 Medline

19. Moller MB. Results of microvascular decompression of the eighth nerve as treatment for disabling positional vertigo. Ann Otol Rhinol Laryngol 1990;99:724-29 CrossRef Medline

20. Clift JM, Wong RD, Carney GM, et al. Radiographic analysis of cochlear nerve vascular compression. Ann Otol Rhinol Laryngol 2009; 118:356-61 CrossRef Medline

21. Hoekstra CE, Prijs VF, van Zanten GA. Diagnostic yield of a routine magnetic resonance imaging in tinnitus and clinical relevance of the anterior inferior cerebellar artery loops. Otol Neurotol 2015;36: 359-65 CrossRef Medline

22. Kim DH, Kim HJ, Hong SK, et al. Neurovascular compression syndrome of the eighth cranial nerve: clinical features and medical treatment. Korean J Otorhinolaryngol Head Neck Surg 2014; 57:518-25 CrossRef

23. Jannetta PJ, Moller MB, Moller AR. Disabling positional vertigo. N Engl J Med 1984;310:1700-05 CrossRef Medline 
24. Reisser C, Schuknecht HF. The anterior inferior cerebellar artery in the internal auditory canal. Laryngoscope 1991;101:761-66 Medline

25. Sirikci A, Bayazit $Y$, Ozer E, et al. Magnetic resonance imaging based classification of anatomic relationship between the cochleovestibular nerve and anterior inferior cerebellar artery in patients with non-specific neuro-otologic symptoms. Surg Radiol Anat 2005;27: 531-35 CrossRef Medline

26. Schick B, Brors D, Koch O, et al. Magnetic resonance imaging in patients with sudden hearing loss, tinnitus and vertigo. Otol Neurotol 2001;22:808-12 CrossRef Medline

27. Song JJ, Vanneste S, Schlee W, et al. Onset-related differences in neural substrates of tinnitus-related distress: the anterior cingulate cortex in late-onset tinnitus, and the frontal cortex in early-onset tinnitus. Brain Struct Funct 2015;220:571-84 CrossRef Medline

28. Song JJ, Vanneste S, De Ridder D. Dysfunctional noise cancelling of the rostral anterior cingulate cortex in tinnitus patients. PLoS One 2015;10:e0123538 CrossRef Medline

29. Song JJ, De Ridder D, Weisz N, et al. Hyperacusis-associated pathological resting-state brain oscillations in the tinnitus brain: a hyper- responsiveness network with paradoxically inactive auditory cortex. Brain Struct Funct 2014;219:1113-28 CrossRef Medline

30. Song JJ, De Ridder D, Schlee W, et al. "Distressed aging": the differences in brain activity between early- and late-onset tinnitus. Neurobiol Aging 2013;34:1853-63 CrossRef Medline

31. Song JJ, Punte AK, De Ridder D, et al. Neural substrates predicting improvement of tinnitus after cochlear implantation in patients with single-sided deafness. Hear Res 2013;299:1-9 CrossRef Medline

32. Kim SH, Jang JH, Lee SY, et al. Neural substrates predicting shortterm improvement of tinnitus loudness and distress after modified tinnitus retraining therapy. Sci Rep 2016;6:29140 CrossRef Medline

33. Elgoyhen AB, Langguth $B$, Nowak W, et al. Identifying tinnitus-related genes based on a side-effect network analysis. CPT Pharmacometrics Syst Pharmacol 2014;3:e97 CrossRef Medline

34. De Ridder D, Heijneman K, Haarman B, et al. Tinnitus in vascular conflict of the eighth cranial nerve: a surgical pathophysiological approach to ABR change. Prog Brain Res 2007;166:401-11 CrossRef Medline 